\title{
Pairwise Coupled Hybrid Vanadium Dioxide-MOSFET (HVFET) Oscillators for Non-Boolean Associative Computing
}

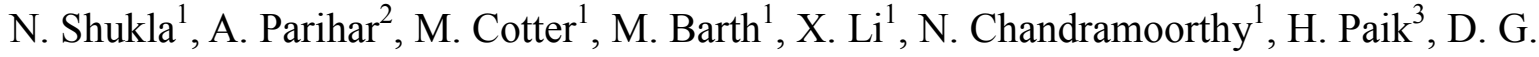 \\ Schlom $^{3}$, V. Narayanan ${ }^{1}$, A. Raychowdhury ${ }^{2}$ and S. Datta ${ }^{1}$ \\ ${ }^{1}$ The Pennsylvania State University, University Park, PA-16801, USA; \\ ${ }^{2}$ Georgia Institute of Technology, Atlanta, Georgia, USA; \\ ${ }^{3}$ Cornell University, Ithaca, NY, USA \\ Phone: (814) 777-8997; Fax: (814) 865-7065; Email: nss152@psu.edu
}

\begin{abstract}
Information processing applications related to associative computing like image / pattern recognition consume excessive computational resources in the Boolean processing framework. This motivates the exploration of a non-Boolean computing approach for such applications. In this work, we demonstrate, (i) novel hybrid set of pair-wise coupled oscillators comprising of vanadium dioxide $\left(\mathrm{VO}_{2}\right)$ metal-insulator-transition (MIT) system integrated with MOSFET; (ii) degree of synchronization between oscillators based on input analog voltage difference; (iii) implementation of hardware platform for fast and efficient evaluation of $\mathrm{L}_{\mathrm{k}}$ fractional distance norm $(\mathrm{k}<1)$; (iv) improved quality of image processing and $\sim 20 \mathrm{X}$ lower power consumption of the coupled oscillators over a CMOS accelerator.
\end{abstract}

\section{Introduction}

Complementary metal-oxide-semiconductor (CMOS) based Boolean logic is the cornerstone of the information technology industry. A class of problems, related to associative processing, requires massive computational resources in the Boolean framework [1]. While this has motivated the development of faster and more efficient algorithms, they are mapped back to the Boolean hardware which ultimately limits their performance. Fundamentally, this "Boolean bottleneck" arises from the requirement of a large number of power-intensive multiply-accumulate (MAC) operations, common to many associative-processing algorithms. This encourages the development of a non-Boolean approach utilizing coupled oscillatory systems (Fig. 1) [2-8]. Here, we experimentally demonstrate for the first time, various degrees of synchronization among hybrid $\mathrm{VO}_{2}$-MOSEFT (HVFET) oscillators in response to an input analog signal difference. The synchronization dynamics enables fast and efficient calculation of a 'fractional distance norm' in the analog domain which is suitable for pattern matching in high dimensional space [9].
Single $\mathrm{VO}_{2}$ Relaxation Oscillator

$\mathrm{VO}_{2}$ is a correlated electron system that exhibits a metalinsulator transition (MIT) with up to 4 orders of abrupt resistivity change in $15 \mathrm{~nm}$ thick epitaxial films (Fig.2b) (bulk films show up to 5 orders). The MIT can be triggered using various external stimuli including electrical triggering [10] (Fig. 2c). This abrupt resistivity change can be engineered into a current controlled negative differential resistance (NDR) regime using an optimum negative feedback to generate sustained charge oscillations [11]. Fig. 3a shows the single $\mathrm{VO}_{2}$ oscillator circuit consisting of a two-terminal $\mathrm{VO}_{2}$ device (Fig. 2a) with a MOSFET in series (HVFET-Oscillator). The details of the $\mathrm{VO}_{2}$ film growth on the $(001) \mathrm{TiO}_{2}$ substrate and the fabrication process are reported elsewhere [12]. All measurements are carried out at $273 \mathrm{~K}$. When the insulator-tometal transition $\left(\mathrm{E}>\mathrm{E}_{2}\right)$ is electrically induced, there is an abrupt change in the conductivity of $\mathrm{VO}_{2}$ (Fig. 2c). The MOSFET (acting like a current source) provides a negative feedback to the $\mathrm{VO}_{2}$ inducing an NDR such that the electric field across $\mathrm{VO}_{2}$ drops below $\mathrm{E}_{1}$ (Fig. $3 \mathrm{~b}$ ). This makes the metallic phase unstable causing $\mathrm{VO}_{2}$ to return to insulating state resulting in a high field drop across $\mathrm{VO}_{2}$ again. The process repeats inducing a stable oscillatory state [11] (Fig. 3c). The operating load line of the oscillator superimposed on the switching characteristics of the $\mathrm{VO}_{2}$ device is illustrated in Fig. $3 \mathrm{~b}$. The oscillation amplitude superimposed on the output characteristics of the MOSFET (Fig. 3d) shows its operating region. Further, an input gate voltage modulates the MOSFET's operating point and enables the HVFET oscillator frequency to be programmed over a decade (Fig. 3e).

\section{Pairwise Coupled Oscillators}

To make such oscillators relevant to computing applications, we explore their synchronization dynamics. Fig. 4a shows the false-colored SEM of the fabricated $\mathrm{VO}_{2}$ devices and illustrates the capacitively coupled oscillator configuration. The high-pass 
t1ltering contiguration tormed by the coupling capacitor $\mathrm{L}_{\mathrm{C}}$ blocks DC interaction while allowing reactive power to couple. The synchronized time domain waveforms are shown in Fig. $4 \mathrm{~b}$ along with the corresponding power spectrum (Fig. $4 \mathrm{c})$. It is clear from Fig. $4 \mathrm{~d}$ that the resonant frequency of the coupled oscillators can be tuned with an input gate voltage difference, $\Delta \mathrm{V}_{\mathrm{GS}}\left(=\mathrm{V}_{\mathrm{GS}, 2}-\mathrm{V}_{\mathrm{GS}, 1}\right)$.

\section{Computing with Coupled HVFET Oscillators}

The nonlinear dynamics of the coupled oscillators are analyzed using the equivalent circuit in Fig. 5. Fig. 6 shows that the relative phase of the two oscillators can be tuned with the gate voltage inputs. The oscillators lock out of phase $\left(\sim 180^{\circ}\right)$ when $\Delta \mathrm{V}_{\mathrm{GS}}=0 \mathrm{~V}$ and the phase difference deviates from this value as $\Delta \mathrm{V}_{\mathrm{GS}}$ increases. The corresponding synchronized oscillator trajectories in phase space can be used to capture the difference between the inputs $\mathrm{V}_{\mathrm{GS}, 1}$ and $\mathrm{V}_{\mathrm{GS}, 2}$. An averaged exclusive-or (XOR) measure is used to analyze the oscillator output. The averaged XOR measure is defined as (i) thresholding the analog output to a binary stream, (ii) applying XOR operation on these binary values at every time instance (iii) averaging this XOR output for a finite time duration. The averaged XOR output is equivalent to the fraction of time the dynamical system spends in the grey region (Fig. 7a) where $\mathrm{XOR}=1$. The XOR output for various input $\mathrm{V}_{\mathrm{GS}}$ values is shown in Fig. 7b. This output of the synchronized oscillators calculates a fractional distance norm, $\left(\mathrm{L}_{\mathrm{k}}\right.$ norm; $\left.\mathrm{k}=0.5\right)$, as seen by its close resemblance to the $\left(x_{i}^{0.5}-y_{i}^{0.5}\right)^{2}$ distance map (Fig. 7c). The experimental and the simulated XOR outputs as a function of input $\Delta \mathrm{V}_{\mathrm{GS}}$ are shown in Fig. 7d, e, respectively.

\section{Associative Processing Application}

We investigate the application of these oscillators for visual saliency approximations (detecting parts of the image that visually standout) [13] (Fig. 8a). Oscillator-based edge detection is performed using an array of pairwise oscillators to approximate the degree of dissimilarity between a given image pixel and its immediate neighbors. Different edges, vertical, horizontal, diagonal, are detected based on the selection of neighboring pixels for comparison. As this concept is expanded to include the comparison of pixels within a larger neighborhood (pixels surrounding reference pixel; a $3 \times 3$ neighborhood is used here), the output approximates the visual saliency (Fig. 8b). It is evident that the oscillators show higher sensitivity to image contrast in comparison to a CMOS ASIC accelerator (Fig. 8a) that uses a linear $\sum\left|\mathbf{x}_{\mathbf{i}}-\mathbf{y}_{\mathbf{i}}\right|$ norm (Fig. $8 b)$.

\section{Power Consumption}

The projected scaling of the input DC voltage and frequency with the $\mathrm{VO}_{2}$ channel length is shown in Fig. 9a,b respectively. Fig. 10a,b shows the pareto chart of the power consumption for each component of the HVFET oscillator

processor and the CMOS ASIC accelerator for visual saliency, respectively. All digital circuits were implemented with $11 \mathrm{~nm}$ node transistor models projected from the $22 \mathrm{~nm}$ node PTM model [14]. The coupled oscillators provide a power reduction of $20 \mathrm{X}$ over CMOS reflecting the advantage of 'let physics do the computing' approach [1] and removing the "Boolean bottleneck".

\section{Conclusions}

In this work, we provide (i) first experimental demonstration of coupled HVFET oscillators with input programmable synchronization; (ii) hardware platform capable of efficiently computing a fractional distance norm and its application in visual saliency; (iii) $\sim 20 \mathrm{X}$ reduction in power dissipation over CMOS.

\section{Acknowledgement}

This work was supported by Office of Naval Research through award N0001411-1-0665. AP was partially funded by a gift from Intel Corporation.. SD and VN acknowledge funding, in part, from the National Science Foundation Expeditions in Computing Award-1317560.

\section{References}

[1] Shibata et al. CNNA, 2012.

[2] Levitan et al. CNNA, 2012.

[3] Narayanan et al. DATE, 2014.

[4] Hoppenstedt et al. PRL, 82,14, 1999.

[4] Datta et al. DAC 2014.

[5] Csaba et al. CNNA, 2012.

[6] Nikonov et al. arXiv: 1304.6125.

[6] Vassilieva et al., IEEE Trans. Neural Netw. 22,84-95 (2011).

[7] Wang et al. IEEE Trans. Neural Netw. 6, 283-6 (1995).

[8] Izhikevich, IEEE Trans. Neural Netw.10, 508-26 (1999).

[9] C. Aggarwal et al. ICDT 200, 2001.

[10] Freeman et al., APL, 103, 26, 2013.

[11] Shukla et al. Sci. Rep, 4, 4964, 2014.

[12] Tashman et al. APL, 104,063104 (2014).

[13] N Bruce PhD thesis, York University, Toronto, 2008.

[14] http://ptm.asu.edu/latest.html. 


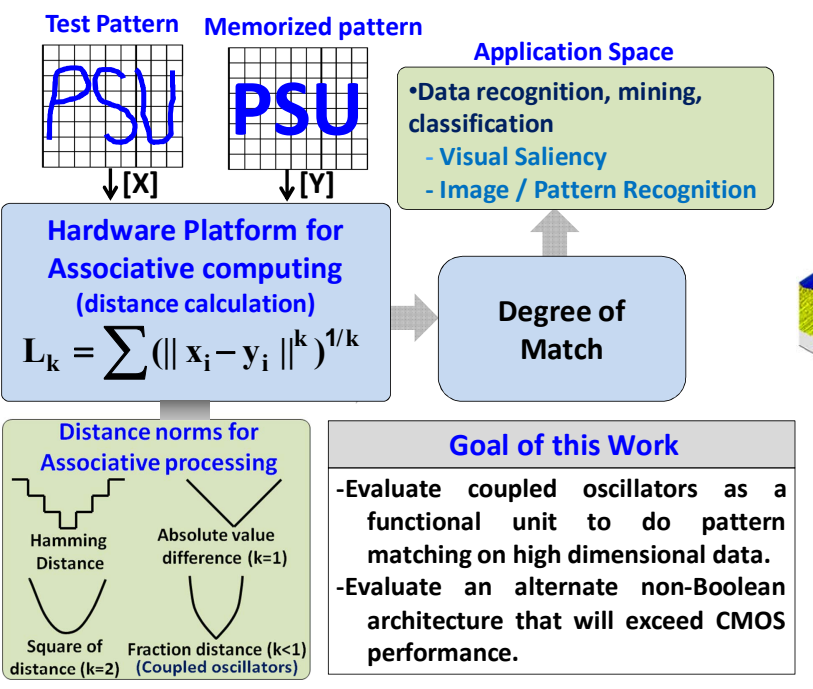

Figure 1| Motivation. We evaluate coupled oscillators as fundamental hardware block for non-Boolean associative computing. The power projection comparison of coupled oscillators with CMOS is also compared.
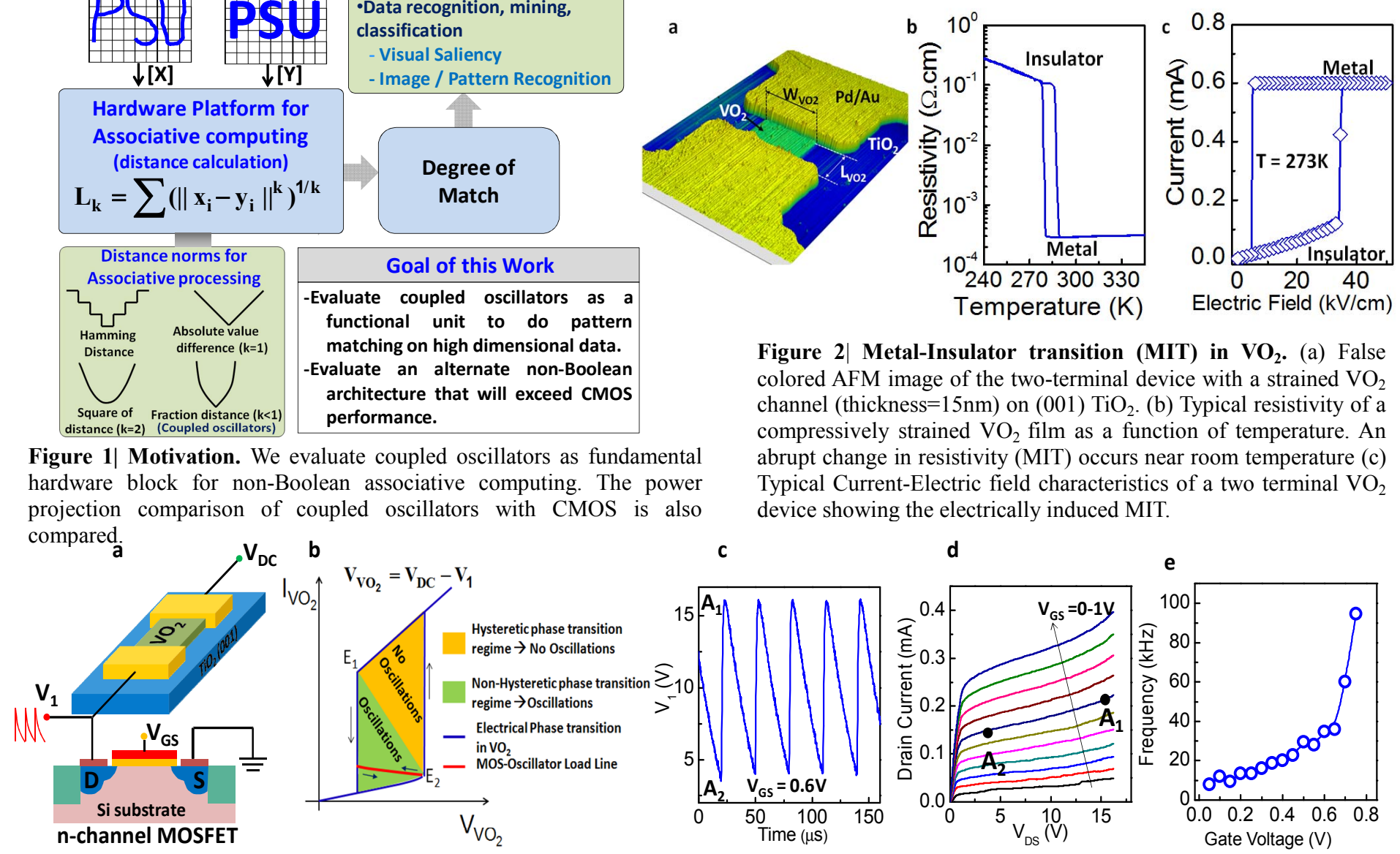

Figure 2| Metal-Insulator transition (MIT) in $\mathbf{V O}_{2}$. (a) False colored AFM image of the two-terminal device with a strained $\mathrm{VO}_{2}$ channel (thickness $=15 \mathrm{~nm}$ ) on $(001) \mathrm{TiO}_{2}$. (b) Typical resistivity of a compressively strained $\mathrm{VO}_{2}$ film as a function of temperature. An abrupt change in resistivity (MIT) occurs near room temperature (c) Typical Current-Electric field characteristics of a two terminal $\mathrm{VO}_{2}$ device showing the electrically induced MIT.

Figure 3| Hybrid VO-MOSFET oscillator (HVFET oscillator) with gate programmable oscillation frequency. (a) Schematic of a VO $\mathrm{VO}_{2}$ relaxation oscillator consisting of a two terminal $\mathrm{VO}_{2}$ device in series with the source-drain of a MOSFET. $\left(\mathrm{L}_{\mathrm{VO} 2}=4 \mu \mathrm{m} ; \mathrm{W}_{\mathrm{VO} 2}=6 \mu \mathrm{m}\right)(\mathrm{b}) \mathrm{Schematic}$ of the operating load line (red) of a HVFET oscillator superimposed on the two terminal I-V characteristics of the $\mathrm{VO}_{2}$ device (blue). The MOSFET induces a non-hysteretic oscillatory regime in $\mathrm{VO}_{2}$ through negative feedback. (c) Time-Domain waveform of the HVFET oscillator. These oscillations have been shown to be stable over $2.5 \times 10^{9}$ cycles. (d) Oscillation amplitude $\left(\mathrm{A}_{1}-\mathrm{A}_{2}\right)$ superimposed on the output characteristics of the MSOFET.(e) Oscillation frequency as a function of gate voltage $\left(\mathrm{V}_{\mathrm{GS}}\right)$ enabling VCO operation.

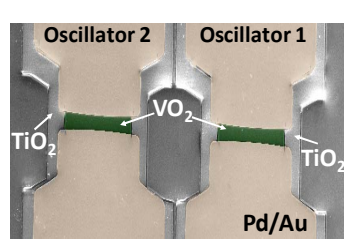

a
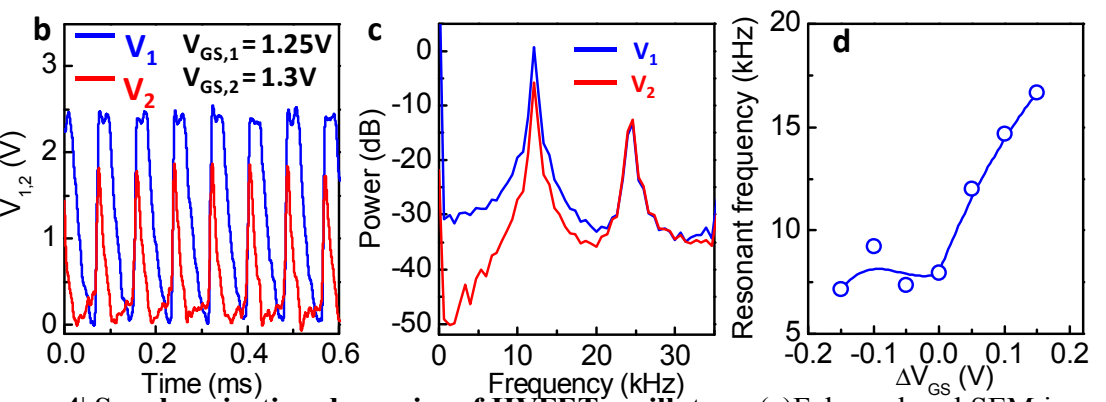

Figure 4 Synchronization dynamics of HVFET oscillators. (a)False colored SEM image of the $\mathrm{VO}_{2}$ devices used to study their synchronization dynamics $\left(\mathrm{L}_{\mathrm{VO} 2}=4 \mu \mathrm{m} ; \mathrm{W}_{\mathrm{VO} 2}=40\right.$ $\mu \mathrm{m})$. Schematic of the capacitively coupled HVFET oscillators $\left(\mathrm{C}_{\mathrm{C}}=2.2 \mathrm{nF}\right)$. (b) Time domain waveform of the synchronized $\mathrm{VO}_{2}$ coupled HVFET oscillator after eliminating DC offsets $\left(\mathrm{V}_{\mathrm{GS}, 1}=1.25 \mathrm{~V}, \mathrm{~V}_{\mathrm{GS}, 2}=1.3 \mathrm{~V}\right)$ (c) Power spectrum of the coupled waveform in (b). (d)

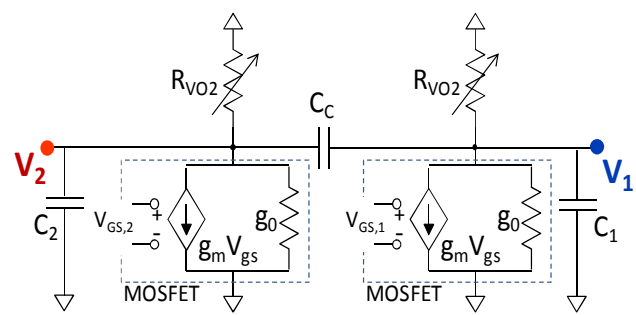

Figure 5| Equivalent circuit of the coupled oscillators. Equivalent circuit of the capacitively coupled oscillators. $\mathrm{C}_{1}$ and $\mathrm{C}_{2}$ are the equivalent capacitance of the $\mathrm{VO}_{2}$ device and the output circuit of the MOSFET.
Variation of coupled frequency as a function of $\Delta \mathrm{V}_{\mathrm{GS}}\left(=\mathrm{V}_{\mathrm{GS}, 2}-\mathrm{V}_{\mathrm{GS}, 1}\right)$

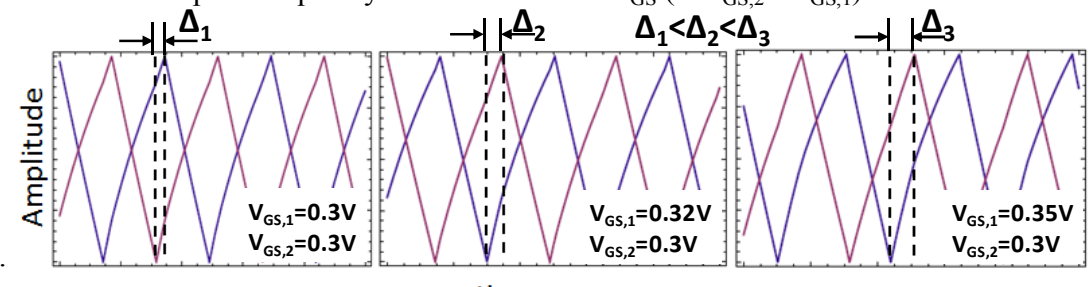

Figure 6| Input gate voltage controlled synchronization characteristics of oscillators. The relative difference (indicated by black lines) between the svnchronized waveforms can be tuned and increases with $\Delta \mathrm{V}_{\text {rs }}$. 

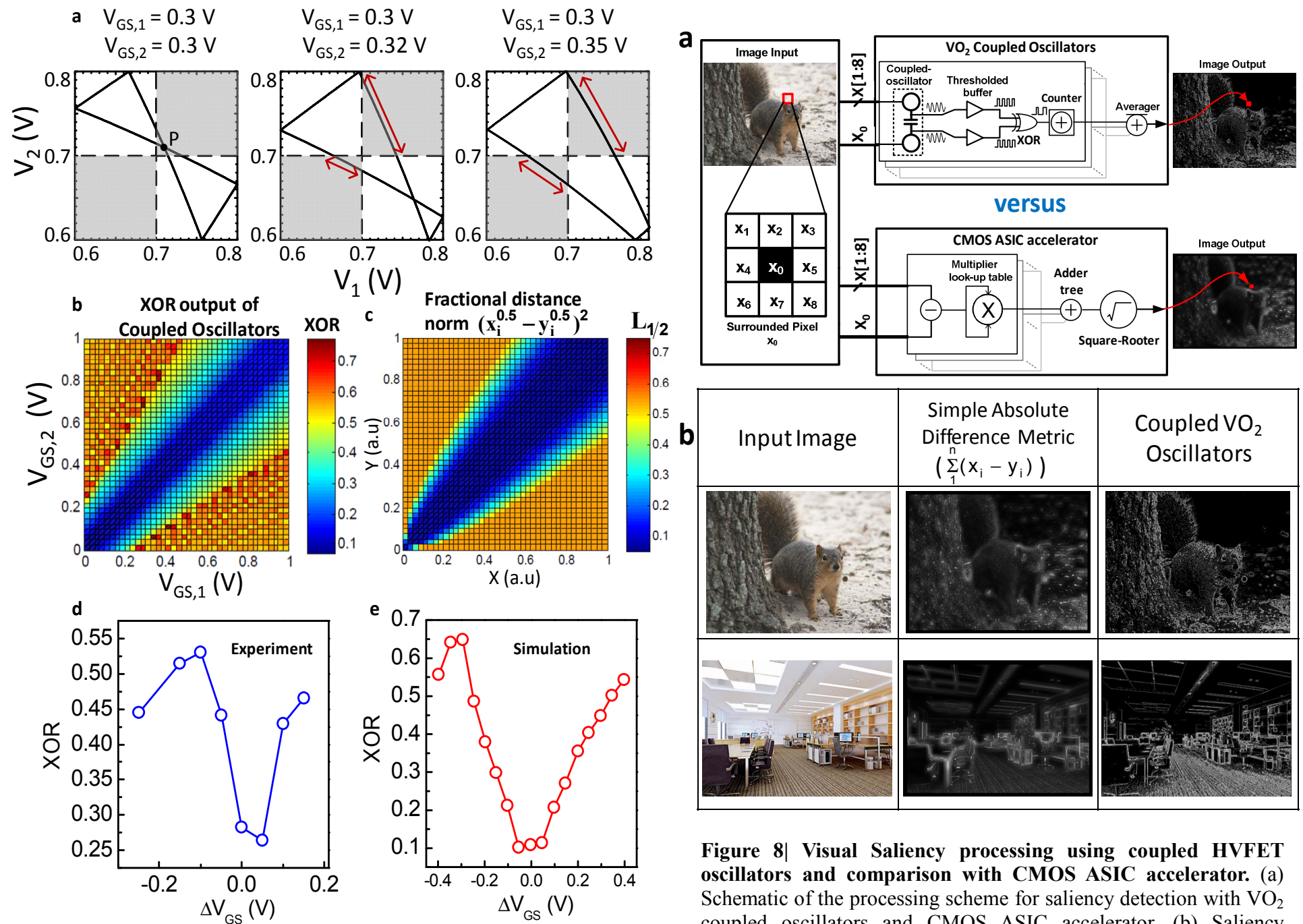

Figure 7| XOR of oscillator output as a fractional distance norm and a computing metric for pair-wise coupled oscillators. (a) Plots showing the relation between averaged XOR output of the oscillators and the steady state periodic orbit of the coupled oscillator system. The averaged XOR output is the fraction of time spent in the gray region, which also captures the phase difference between $V_{\mathrm{GS}, 1}$ and $\mathrm{V}_{\mathrm{GS}, 2}$. (b) Simulated XOR output as a function of $\mathrm{V}_{\mathrm{GS}, 1}$ and $\mathrm{V}_{\mathrm{GS}, 2}$. The $\mathrm{XOR}$ value is minimum when $\mathrm{V}_{\mathrm{GS}, 1}=\mathrm{V}_{\mathrm{GS}, 2}$. (c) Fractional distance norm $\left(\mathrm{x}_{\mathrm{i}}^{0.5}-\mathrm{y}_{\mathrm{i}}^{0.5}\right)^{2}$. The $\mathrm{XOR}$ metric computes the Euclidean distance as seen by the similarity between the (b) and (c). (d) Experimental (e) Simulated XOR as a function of $\Delta \mathrm{V}_{\mathrm{GS}}$.
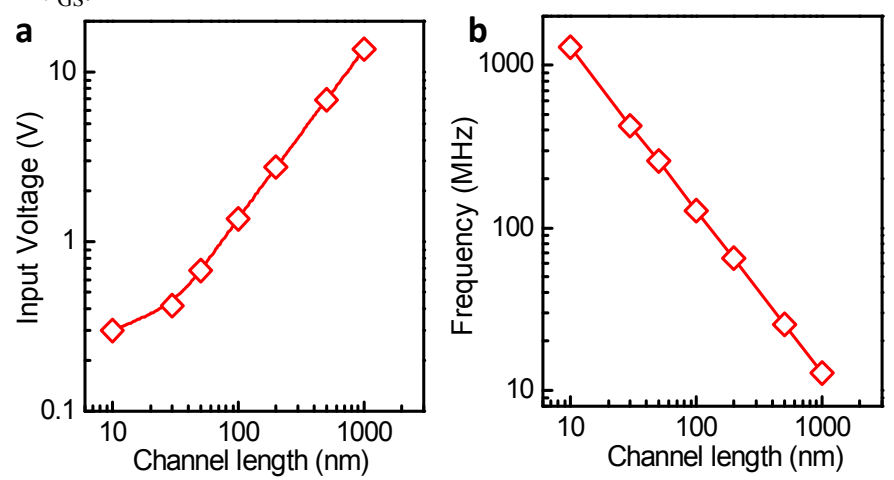

Figure 9| Projected $\mathrm{VO}_{2}$ oscillator scaling. (a) Scaling of input DC voltage of the oscillator (b) Projected scaling of oscillator frequency with the $\mathrm{VO}_{2}$ channel length.

Figure 8| Visual Saliency processing using coupled HVFET oscillators and comparison with CMOS ASIC accelerator. (a) Schematic of the processing scheme for saliency detection with $\mathrm{VO}_{2}$ coupled oscillators and CMOS ASIC accelerator. (b) Saliency detection outputs obtained with $\mathrm{VO}_{2}$ coupled oscillators and using the simple $\sum_{1}^{\mathrm{n}}\left(\mathrm{x}_{\mathrm{i}}-\mathrm{y}_{\mathrm{i}}\right)$ metric.

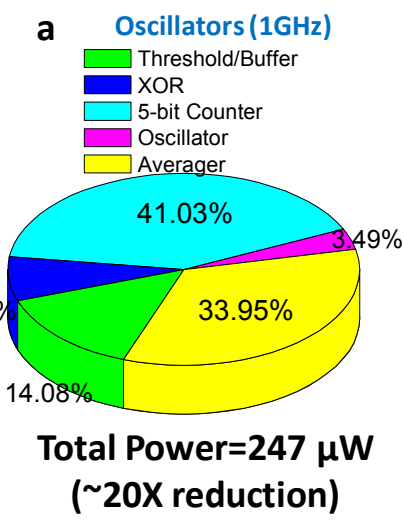
b $\quad 11 \mathrm{~nm}$ node CMOS Accelerator
$\square$ Adder Tree
Square-Rooter $\square$ ADC Converter $\square$ Control Logic Other Computation (includin

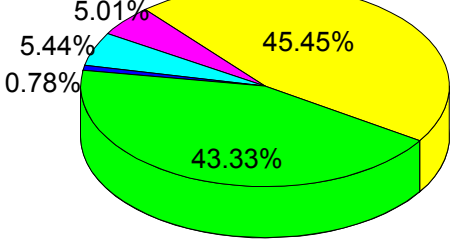

Total Power=5.138 $\mathrm{mW}$

Figure 10| Power comparison. (a) Power dissipation break-down of individual components in the oscillatory processing scheme. (b) Power dissipation breakdown of individual components in a conventional synthesized CMOS ASIC processing accelerator. The Adder tree and the MAC instructions contribute to the bottle-neck in this Boolean processing scheme. All the digital circuits were implemented with $11 \mathrm{~nm}$ node transistor models projected from the 22nm PMT model. 